\section{Prevalência de internação hospitalar e fatores associados: um estudo de base populacional em um centro urbano no Sul do Brasil}

\author{
Prevalence and factors associated with hospital \\ admissions in a population-based study in a \\ southern Brazilian city
}

\author{
1 Programa de Pós-graduação \\ em Saúde Coletiva, \\ Universidade Luterana do \\ Brasil, \\ Canoas, Brasil. \\ Correspondência \\ J. U. Béria \\ Programa de Pós-graduação \\ em Saúde Coletiva \\ Universidade Luterana do \\ Brasil. \\ Av. Farroupilha 801 , \\ Canoas, $R S$ \\ 92420-280, Brasil. \\ jberia@terra.com.br
}

\begin{abstract}
This cross-sectional study investigates the use of health services and associated factors in individuals $\geq 14$ years of age in Canoas, Rio Grande do Sul, Brazil. 1,954 persons were interviewed in 40 census tracts. One-year prevalence of hospital admissions was 9.4\%. Adjusted data analysis showed that hospitalization was associated with: age $\geq 60$ years $(R P=4.14 ; 95 \%$ CI: 2.07-8.25), physician visit in the previous two months ( $R P=2.79$; 95\%CI: 2.03-3.83), and $\geq 2$ stressful life events ( $R P=1.83$; 95\%CI: 1.19-2.80). Individual income of $\geq 2.10$ times the prevailing minimum wage was associated with decreased likelihood of hospitalization $(R P=0.60 ; 95 \%$ CI: 0.41-0.87). Prevalence of hospital admissions was consistent with other studies. Higher prevalence of hospitalization in lower-income groups may indicate decreased access to primary health care. Other possible factors are higher morbidity and severity of diseases among lower-income groups. Future research should focus on the relationship between morbidity and stressful life events.
\end{abstract}

Hospital Services; Hospitalization; Stressful Events

\author{
Ligia Regina Garbinato 1 \\ Jorge Umberto Béria 1 \\ Andréia Cristina Leal Figueiredo 1 \\ Beatriz Raymann 1 \\ Luciana Petrucci Gigante 1 \\ Lilian dos Santos Palazzo 1 \\ Denise Rangel Ganzo de Castro Aerts
}

\section{Introdução}

O estudo do padrão de utilização de serviços de saúde pela população é indispensável para o planejamento e desenvolvimento de ações que busquem reduzir as desigualdades no adoecer e no acesso aos serviços de saúde 1,2.

O tema utilização de serviços de saúde compreende diferentes desfechos, incluindo consultas médicas curativas, preventivas e internações. Tem-se mostrado crescente atenção para a assistência hospitalar devido ao papel fundamental nos serviços de saúde e um custo muito elevado. Da mesma forma o entendimento dos fatores associados à utilização de serviços hospitalares é essencial para a discussão de políticas de saúde.

Um estudo de base populacional em um centro urbano no Sul do país, em 1992, encontrou uma prevalência de internações de $9 \%$ para a população adulta ${ }^{3}$. Outro estudo que utilizou dados do ano de 1998 da Pesquisa Nacional de Amostra por Domicílios (PNAD) trouxe resultado inferior (6,9\%), porém não teve restrições para idade 4 . Neste último estudo também foram observados os fatores associados ao desfecho, demonstrando uma maior utilização dos serviços hospitalares por indivíduos do sexo feminino $(8,7 \%)$, grupo etário de oitenta anos ou mais $(18,7 \%)$, com maiores necessidades em saúde, menor renda e baixa escolaridade (quando ajusta-se para os fatores facilitadores do acesso) 4 . 
O presente artigo fundamenta-se em um levantamento de base populacional, realizado em Canoas, Rio Grande do Sul, Brasil, e tem por objetivo estudar a prevalência de internações hospitalares na população de 14 anos ou mais em Canoas, no período de um ano, analisando também as associações deste evento com fatores demográficos, sócio-econômicos, utilização de serviços de saúde, eventos estressantes e percepção de saúde.

\section{Material e método}

Este trabalho está integrado a um estudo mais amplo sobre a saúde da população da cidade de Canoas. Foi realizado um estudo transversal, com base populacional, no período de novembro de 2002 a junho de 2003. A população-alvo foi composta por residentes da zona urbana da cidade de Canoas, Região Metropolitana de Porto Alegre, Rio Grande do Sul, no Sul do Brasil.

A cidade de Canoas possui 391 setores censitários. Destes, foram sorteados aleatoriamente quarenta setores. Foram visitados 26 domicílios por setor censitário. Em cada setor, a partir de um quarteirão previamente sorteado, foi escolhida também, de maneira aleatória, a esquina que seria o ponto de partida. A partir dessa esquina, à esquerda do entrevistador em frente da casa de número um, visitou-se a quarta casa, a oitava e a décima segunda casa, sistematicamente.

Utilizou-se um questionário padronizado, pré-codificado e testado previamente, aplicado por duplas compostas de um estudante do curso de medicina e de uma fonoaudióloga, previamente submetidos a programa de treinamento e estudo piloto.

Os questionários foram codificados e posteriormente submetidos à dupla digitação. A entrada e a edição dos dados foram efetuadas com o auxílio do Programa USCA (Utilização de Serviços em Canoas), especialmente desenvolvido para o estudo.

O banco de dados resultante registrou 2.609 indivíduos de todas as faixas etárias, apresentando uma perda de $21 \%$, especialmente entre os homens de 25 a 49 anos.

O presente estudo selecionou como população-alvo indivíduos com idade de 14 anos ou mais, incluindo, portanto, um total de 1.954 pessoas, com perda de $20,3 \%$. Por tratar-se de um estudo satélite, o seu poder estatístico foi calculado posteriormente com o programa Power 5, tendo-se obtido um resultado de $90 \%$ para todas as associações.

As variáveis utilizadas no estudo foram: (1) variável dependente: hospitalização no último ano; (2) variáveis independentes: demográficas (idade, sexo e situação conjugal), sócio-econômicas (renda e escolaridade), de necessidade em saúde (auto-avaliação de saúde - utilizou-se três categorias: estado de saúde bom, regular e ruim), referentes aos serviços de saúde (ter ou não acesso a seguro privado de saúde e realização de consulta médica nos últimos dois meses antes da entrevista) e eventos estressantes (separação conjugal, assalto/roubo, perda do emprego, abuso sexual e maltrato físico).

Os dados foram analisados pelo programa Stata 8.0 (Stata Corporation, College Station, Estados Unidos) utilizando-se procedimentos estatísticos padronizados.

A análise estatística foi feita em três etapas: (1) análise descritiva (univariada), incluindo a freqüência de cada variável do estudo; (2) análise bivariada, entre a variável dependente e as variáveis independentes, aplicação do teste qui-quadrado, resultando na determinação da razão de prevalência (RP) e respectivo intervalo de confiança de $95 \%$ para cada característica da população estudada; (3) análise multivariada, utilizou o método de regressão de Poisson incluindo somente as variáveis associadas ao desfecho em um nível de significância menor ou igual a 0,2. Essa análise foi feita por níveis de acordo com o modelo de análise hierarquizado (Figura 1), embasado no estudo de MendozaSassi \& Béria ${ }^{6}$. Quando da inclusão de novas variáveis, a cada nível, utilizou-se o método $b a$ ckward, permanecendo no modelo as variáveis associadas ao desfecho em um nível de significância menor que 0,05 , no teste de razão de verossimilhança. A análise foi ajustada para efeito de delineamento.

Esta pesquisa foi aprovada pelo Comitê de Ética em Pesquisa em Seres Humanos e Animais da Universidade Luterana do Brasil, conforme termo de aprovação n. 2004/078H.

\section{Resultados}

A prevalência geral de internação encontrada na população, no período de um ano, foi de 9,4\%. Pouco mais da metade dos indivíduos eram do sexo feminino $(57,4 \%)$. As faixas etárias relativas aos intervalos de 20 a 39 anos e de 40 a 59 anos apresentaram maior proporção de indivíduos: $38,7 \%$ e $34,5 \%$, respectivamente. Das pessoas entrevistadas, $17,5 \%$ possuíam escolaridade de três anos ou menos, e, destas, 3,9\% eram analfabetas. A renda individual de mais de dois salários mínimos (R\$ 400,00 - salário mínimo nacional referente a maio de 2003) foi a mais freqüente (40,6\%). 
Modelo de análise de utilização de serviços hospitalares.
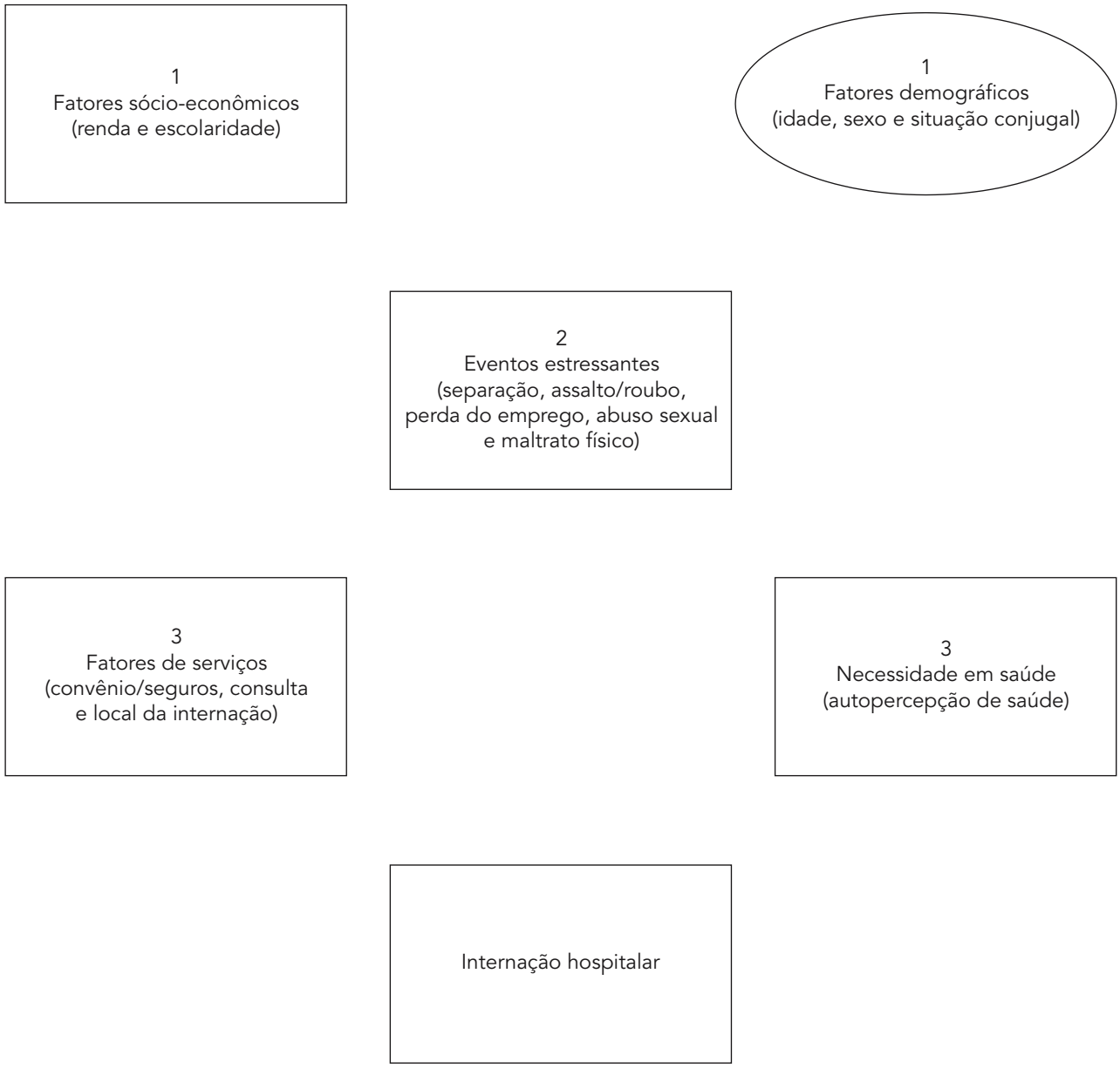

A maioria $(67,5 \%)$ não referia nenhum dos eventos estressantes estudados e considerava seu estado de saúde como bom $(63,2 \%)$.

Dentre osindivíduos amostrados, 44,6\% informaram ter consultado o médico, ao menos uma vez, nos dois meses anteriores à entrevista e, destes, $15,1 \%$ também internaram no período de um ano. Entre os indivíduos que internaram, menos da metade (47\%) o fizeram na cidade de Canoas, a maioria das internações ocorreu em Porto Alegre $(49,7 \%)$ e apenas $3,3 \%$ em outras localidades.

As prevalências de internação foram maiores entre indivíduos do sexo feminino (11,1\%), em comparação com os do sexo masculino $(7,1 \%)$. A faixa etária de sessenta anos ou mais apresentou a maior prevalência de internação $(17,2 \%)$ e a de 14-19 anos, a menor (3,5\%). Os indivíduos que vi- venciaram dois ou mais eventos estressantes e os que referiram um estado de saúde ruim, tiveram uma prevalência de internação superior a $15 \%$.

A RP bruta encontrada para internação hospitalar e para os demais fatores em estudo está disposta na Tabela 1. Foi possível verificar que, entre os fatores demográficos, o sexo feminino apresentou $55 \%$ mais chances de internação quando comparado ao sexo masculino. E, como esperado, dentre as faixas etárias estudadas, a de sessenta anos ou mais demonstrou ter uma chance de internação 4,96 vezes mais que a faixa etária utilizada como referência (14-19 anos).

Com relação aos fatores sócio-econômicos, verificou-se que um maior número de anos de estudo (oito anos ou mais) foi um fator protetor para internação $(\mathrm{RP}=0,56)$. Indivíduos com ren- 
Tabela 1

Distribuição de internação hospitalar segundo fatores demográficos, sócio-econômicos, eventos estressantes e auto-avaliação de saúde.

Canoas, Rio Grande do Sul, Brasil, 2003.

\begin{tabular}{|c|c|c|c|c|c|}
\hline \multirow[b]{2}{*}{ Características } & \multicolumn{2}{|c|}{ Internação } & \multirow[b]{2}{*}{ RP bruta } & \multirow[b]{2}{*}{ IC95\% } & \multirow[b]{2}{*}{$\mathrm{p}$} \\
\hline & $\mathrm{n}$ & $\%$ & & & \\
\hline Sexo & & & & & 0,003 \\
\hline Masculino & 124 & 11,1 & 1,00 & & \\
\hline Feminino & 59 & 7,1 & 1,55 & $1,16-2,09$ & \\
\hline Situação conjugal & & & & & 0,253 \\
\hline Com companheiro & 118 & 10,0 & 1,00 & & \\
\hline Sem companheiro & 63 & 8,5 & 0,84 & $0,63-1,12$ & \\
\hline Faixa etária (anos) & & & & & 0,0001 \\
\hline 14-19 & 9 & 3,5 & 1,00 & & \\
\hline $20-39$ & 71 & 9,4 & 2,72 & $1,37-5,37$ & \\
\hline $40-59$ & 58 & 8,6 & 2,48 & $1,25-4,90$ & \\
\hline 60 ou mais & 45 & 17,2 & 4,96 & $2,47-9,93$ & \\
\hline Analfabeto & & & & & 0,252 \\
\hline Não & 10 & 13,2 & 1,00 & & \\
\hline Sim & 173 & 9,2 & 1,42 & $0,78-2,59$ & \\
\hline Escolaridade (anos completos) & & & & & 0,013 \\
\hline 11 ou mais & 46 & 13,5 & 0,56 & $0,38-0,83$ & \\
\hline $8-10$ & 60 & 9,9 & 0,56 & $0,36-0,87$ & \\
\hline $4-7$ & 30 & 7,6 & 0,73 & $0,51-1,04$ & \\
\hline 3 ou menos & 47 & 7,7 & 1,00 & & \\
\hline Renda individual (salários mínimos) * & & & & & 0,0058 \\
\hline 2,10 ou mais & 63 & 9,7 & 0,74 & $0,52-1,05$ & \\
\hline $0,76-2,00$ & 63 & 12,5 & 1,28 & $0,93-1,77$ & \\
\hline $0,00-0,75$ & 57 & 7,2 & 1,00 & & \\
\hline Convênio & & & & & 0,359 \\
\hline Sim & 98 & 8,8 & 1,00 & & \\
\hline Não & 83 & 10,0 & 1,13 & $0,86-1,50$ & \\
\hline Eventos estressantes ${ }^{\star \star}$ & & & & & 0,0163 \\
\hline Nenhum & 106 & 8,4 & 1,00 & & \\
\hline Um & 49 & 10,5 & 1,24 & $0,89-1,73$ & \\
\hline Dois ou mais & 22 & 15,5 & 1,84 & $1,21-2,80$ & \\
\hline Estado de saúde auto-avaliado & & & & & 0,003 \\
\hline Bom & 95 & 7,7 & 1,00 & & \\
\hline Regular & 73 & 11,7 & 1,51 & $1,13-2,01$ & \\
\hline Ruim & 14 & 15,4 & 1,99 & $1,17-3,39$ & \\
\hline 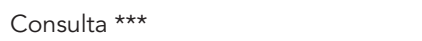 & & & & & 0,0001 \\
\hline Não & 131 & 15,1 & 1,00 & & \\
\hline Sim & 51 & 4,7 & 3,19 & $2,34-4,36$ & \\
\hline
\end{tabular}

* $\mathrm{R} \$ 200,00$;

** Assalto, separação, perda de emprego, maltrato físico, abuso sexual;

*** Consulta nos últimos dois meses.

da individual de 2,10 salários mínimos ou mais tiveram menos chances de internação hospitalar (RP = 0,74; IC95\%: 0,52-1,05), quando comparados aos indivíduos que possuem renda inferior a 0,75 salário mínimo.

Salienta-se a relação apresentada entre a variável eventos estressantes e o desfecho: comparando os indivíduos que referiram não ter sofrido nenhum evento estressante àqueles que referiram um evento, os últimos tiveram um aumento de $24 \%$ da chance de internação (apesar do IC95\% ter englobado a unidade, o teste para tendência linear foi significativo); enquanto que os indivíduos que sofreram dois ou mais eventos apresentaram $84 \%$ mais chances de ocorrência do desfecho. 
Ressalta-se, ainda, que indivíduos com estado de saúde auto-avaliado como ruim tiveram $51 \%$ mais chances de apresentar o desfecho, quando comparados àqueles que referiram um estado de saúde bom. A chance de internação dos indivíduos que consultaram nos últimos dois meses foi três vezes superior a dos que não consultaram.

Os resultados finais da análise multivariada de fatores associados com a ocorrência de hospitalizações no último ano estão dispostos na Tabela 2. Das variáveis pertencentes ao primeiro nível do modelo de análise (demográficas e sócio-econômicas) permaneceram associadas de forma significativa ao desfecho apenas a faixa etária e a renda individual, não demonstrando grandes diferenças na RP ajustada quando comparadas com a RP bruta.

A relação encontrada na variável eventos estressantes permaneceu após o ajustamento para as demais variáveis (sexo, idade, escolaridade, renda individual, consulta médica e auto-avaliação de saúde). Indivíduos que enfrentaram dois ou mais eventos considerados estressantes tive- ram uma chance de $83 \%$ a mais de internar do que indivíduos sem nenhum evento.

A variável consulta também permaneceu após a análise ajustada para as demais variáveis, sugerindo que indivíduos que consultaram no período de dois meses anteriores à entrevista tiveram 2,79 vezes mais chances de internar, quando comparados aos que não consultaram no mesmo período.

\section{Discussão}

O presente estudo tem delineamento transversal, em decorrência disso os resultados obtidos devem ser vistos como fatores associados. É importante salientar, também, que a pesquisa apresentou um maior número de perdas entre os homens de 25 a 49 anos, o que pode ter afetado os resultados, levando a uma superestimação da prevalência geral de internação, pois esta apresentou associação com o sexo feminino. Entre os fatores que levaram ao elevado número de perdas salienta-se: (1) necessidade de assinar um

Tabela 2

Internação hospitalar de acordo com variáveis sócio-econômicas, demográficas, eventos estressantes e consulta média, análise multivariada. Canoas, Rio Grande do Sul, Brasil, 2003

\begin{tabular}{|c|c|c|c|c|c|}
\hline Características & $\mathbf{n}$ & $\%$ & $\begin{array}{l}\text { RP bruta } \\
\text { (IC95\%) }\end{array}$ & $\begin{array}{l}\text { RP ajustada } \\
\text { (IC95\%) }\end{array}$ & $\mathrm{p}$ \\
\hline \multicolumn{6}{|c|}{ Renda individual (salários mínimos) *, } \\
\hline 2,10 ou mais & 794 & 7,2 & $0,74(0,52-1,05)$ & $0,60(0,41-0,87)$ & \\
\hline $0,76-2,00$ & 508 & 12,5 & $1,28(0,93-1,77)$ & $0,92(0,65-1,29)$ & 0,005 \\
\hline $0,00-0,75$ & 652 & 9,7 & 1,00 & 1,00 & \\
\hline \multicolumn{6}{|c|}{ Faixa etária (anos) ** } \\
\hline $14-19$ & 261 & 3,5 & 1,00 & & \\
\hline $20-39$ & 756 & 9,4 & $2,72(1,37-5,37)$ & $2,54(1,27-5,06)$ & \\
\hline $40-59$ & 675 & 8,6 & $2,48(1,25-4,90)$ & $2,46(1,25-4,81)$ & 0,0001 \\
\hline 60 ou mais & 262 & 17,2 & $4,96(2,47-9,93)$ & $4,14(2,07-8,25)$ & \\
\hline \multicolumn{6}{|c|}{ 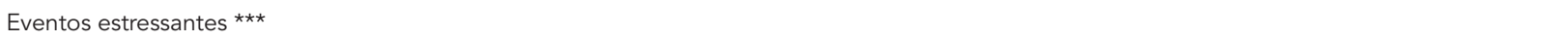 } \\
\hline Nenhum & 1.267 & 8,4 & 1,00 & & \\
\hline Um & 469 & 10,5 & $1,24(0,89-1,73)$ & $1,29(0,93-1,78)$ & 0,018 \\
\hline Dois ou mais & 142 & 15,5 & $1,84(1,21-2,80)$ & $1,83(1,19-2,79)$ & \\
\hline \multicolumn{6}{|l|}{ Consulta \#,\#\# } \\
\hline Não & 1.078 & 4,7 & 1,00 & & 0,0001 \\
\hline $\operatorname{Sim}$ & 868 & 15,1 & $3,19(2,34-4,36)$ & $2,79(2,03-3,83)$ & \\
\hline
\end{tabular}

* R\$ 200,00;

** Primeiro nível (sexo, faixa etária, renda individual e escolaridade);

*** Segundo nível (primeiro nível + eventos estressantes);

\# Terceiro nível (segundo nível + consulta médica e auto-avaliação de saúde);

\#\# Consulta nos últimos dois meses. 
consentimento (estudos populacionais utilizando consentimento verbal costumam ter perdas menores) e (2) em alguns setores censitários sorteados houve dificuldade de acesso dos entrevistadores devido à violência.

A prevalência de internações hospitalares para o período estudado foi de $9,4 \%$ em Canoas, sendo, portanto, muito próxima ao porcentual de $9 \%$ encontrado em estudo realizado no Sul do Brasil em 1992 3, porém, superior ao coeficiente de 6,9\% encontrado para o Brasil (sem restrições de idade), no estudo de Castro et al. ${ }^{4}$, com base em dados do ano de 1998.

Indivíduos com sessenta anos ou mais continuaram fortemente associados ao desfecho, mesmo na análise da RP ajustada ( $R P=4,14$; IC95\%: $2,07-8,25)$. Esse fato reforça dados encontrados na literatura referente ao assunto $4,6,7$. Considerando-se que a população idosa vem crescendo de forma rápida no Brasil nos últimos anos 8 , a relevância do resultado anteriormente descrito está justamente em alertar os gestores de saúde pública para a necessidade de reorganizar e aprimorar os sistemas de atenção à saúde, a fim de que se obtenha um atendimento satisfatório dessa demanda.

A relação existente entre a situação financeira e a internação hospitalar foi analisada a partir da renda individual. Sabe-se que o uso dessa variável possibilita a ocorrência de distorções de resultado com relação aos indivíduos que não possuem renda e dependem do chefe da família. Porém, esse foi o dado coletado (com relação à renda) que sofreu o menor número de perdas.

A morbidade referida cresce na população na proporção inversa da renda 9,10. Os estudos de Almeida et al. 11 e Travassos et al. 12 afirmam que as desigualdades observadas nesse tipo de serviço (internações), no Brasil, são mais sutis que aquelas observadas no consumo geral de serviços de saúde.

Porém, deve-se considerar que o fato de uma maior utilização de serviços hospitalares encontrar-se ligada à população com renda individual entre 0,76 e 2 salários mínimos, pode estar relacionado ao modelo de atenção vigente. Isso porque, ainda que se idealize um modelo de atenção à saúde, com base na promoção da saúde, sabe-se que o Sistema Único de Saúde (SUS) ainda continua, de forma mais intensa, atuando e organizando ações de saúde com vistas a atender aqueles que procuram por assistência médica (ambulatórios, centros de saúde) e hospitalar (urgência e emergência), não tendo uma implantação satisfatória em termos de atendimento na rede básica. Em decorrência disso, a assistência hospitalar tem sido uma porta de entrada para o SUS, favorecendo um maior número de interna- ções que poderiam ser evitadas, caso houvesse uma melhor prática da atenção primária à saúde. Outra questão a ser considerada, refere-se à chamada "internação social", que acontece devido às dificuldades econômicas e sociais do paciente, que é internado para ter acesso ao tratamento adequado (viés de admissão). Outros fatores para a maior hospitalização nos grupos de menor nível sócio-econômico poderiam ser a maior morbidade e severidade da doença entre os grupos mais pobres.

Salienta-se o fato de que menos da metade das internações hospitalares ocorre na cidade de Canoas, o que demonstra que a capacidade instalada de leitos da cidade é insuficiente.

Estudo realizado em 1992 mostrou existir uma relação positiva entre a internação hospitalar no último ano e a freqüência de consultas médicas ${ }^{3}$. O presente estudo encontrou uma associação positiva entre essa variável e o desfecho. Sabe-se, entretanto, que os usuários do SUS ainda encontram dificuldades para conseguir consultas com especialistas e, principalmente, para ter acesso a exames complementares, o que também pode contribuir para um aumento nas internações, pois estas representam uma forma de se conseguir realizar exames de alta complexidade e também de ter a assistência do médico especialista durante o período de internação. Assim, acredita-se ser necessário um aprimoramento do nível de atendimento ambulatorial, de modo a alcançar a idealizada integralidade de atenção, que tende a ter, por conseqüência, a redução dos índices de internação hospitalar.

A associação entre percepção de saúde ruim e internação hospitalar não permaneceu após a análise multivariada, o que pode estar ligado à percepção de saúde dos indivíduos com menor renda, porque é possível que essas pessoas declarem estado de saúde ruim com menos freqüência. Da mesma forma, a variável possuir seguro de saúde privado não manteve associação, pois alguns dos seguros locais de saúde não cobrem internação hospitalar.

Os resultados da análise multivariada indicam uma relação dose-resposta onde indivíduos com maior número de eventos estressantes têm uma chance maior de internação hospitalar. Resultado semelhante já foi encontrado em um estudo realizado no Sul do Brasil, porém, com relação à utilização de serviços ambulatoriais 13 . Segundo um estudo da Organização Mundial da Saúde (OMS), o evento estressante abuso sexual tem um papel importante na carga global das doenças. Esse mesmo estudo salienta a necessidade de estudos em nível micro de fatores de risco como, por exemplo, eventos estressantes e a carga regional de doenças 14 . Assim, seria im- 
portante desenvolver investigações sobre a relação entre eventos estressantes e morbidade, para esclarecer se estes são marcadores de risco ou determinantes.

Saúde encontra-se intimamente ligada à qualidade de vida e, admitindo-se as dificuldades típicas do ambiente urbano em um país com tantas desigualdades, enfatiza-se a importância do movimento que incentiva a promoção da saúde por meio de ações que busquem melhorar as condições de vida da população. Assim, salienta-se a importância da formulação e implementação de políticas sociais que apresentem resultados práticos para a população com relação à segurança, à cultura, à educação, ao lazer, à interação social e às oportunidades de trabalho, com significante incremento nos níveis de qualidade de vida e de saúde dos cidadãos.

\section{Resumo}

Este estudo transversal investiga a utilização de serviços hospitalares e fatores associados em indivíduos com 14 anos ou mais em Canoas, Rio Grande do Sul, Brasil. Foram entrevistados 1.954 indivíduos de 40 setores censitários. A prevalência de internação hospitalar no período de um ano foi de 9,4\%. Na análise ajustada para as demais variáveis, as que permaneceram associadas a uma maior chance de hospitalização foram: idade de 60 anos ou mais $(R P=4,14$; IC95\%: 2,07-8,25), realização de consulta médica nos últimos dois meses ( $R P=2,79$; IC95\%: 2,03-3,83), a ocorrência de dois ou mais eventos estressantes $(R P=1,83$; IC95\%: 1,19-2,80). A renda individual, de 2,10 salários mínimos ou mais, esteve associada a uma menor chance de hospitalização (RP = 0,60; IC95\%: 0,41-0,87). A prevalência de internações encontrada é compatível com outros estudos. A maior prevalência de hospitalização nos grupos de menor nível sócio-econômico pode indicar um menor acesso aos serviços de atenção básica. Outros fatores envolvidos poderiam ser a maior morbidade e severidade da doença entre os grupos mais pobres. Salienta-se a importância de investigar a relação entre eventos estressantes e morbidade.

Serviços Hospitalares; Hospitalização; Eventos Estressantes

\section{Colaboradores}

L. R. Garbinato participou da análise dos dados e redação do artigo. J. U. Béria, B. Raymann e L. P. Gigante participaram da coordenação geral do projeto que deu origem ao levantamento de base populacional, que subsidiou este estudo e redação do artigo. A. C. L. Figueiredo coordenou o trabalho de campo e tratamento dos dados do levantamento de base populacional que subsidiou este estudo, e colaborou na análise de dados e redação do artigo. L. P. Gigante participou da coordenação do trabalho de campo e tratamento dos dados. L. S. Palazzo foi responsável pelo estudo dos eventos estressantes. D. R. G. C. Aerts contribuiu como responsável pela auto-avaliação de saúde.

\section{Agradecimentos}

Este estudo faz parte de um estudo mais amplo que contou com apoio de Christoffel Blindenmission, Fundação de Amparo à Pesquisa do Estado do Rio Grande do Sul e Conselho Nacional de Desenvolvimento Científico e Tecnológico. 


\section{Referências}

1. Fernández de la Hoz K, Leon DA. Self-perceived health status and inequalities in use of health services in Spain. Int J Epidemiol 1996; 25:593-603.

2. Hulka BS, Wheat JR. Patterns of utilization: the patient perspective. Med Care 1985; 23:438-60.

3. Costa JD, Facchini LA. Utilização de serviços ambulatoriais em Pelotas: onde a população consulta e com que freqüência. Rev Saúde Pública 1997; 31:360-9.

4. Castro MSM, Travassos C, Carvalho MS. Fatores associados às internações hospitalares no Brasil. Ciênc Saúde Coletiva 2002; 7:795-811.

5. Dupont WD, Plummer WD. Power and sample size calculation: a review and computer program. Control Clin Trials 1990; 11:116-28.

6. Mendoza-Sassi R, Béria JU. Utilización de los servicios de salud: una revisión sistemática sobre los factores relacionados. Cad Saúde Pública 2001; 17:819-32.

7. Fernández-Mayoralas G, Rodríguez V, Rojo F. Health services accessibility among Spanish elderly. Soc Sci Med 2000; 50:17-26.

8. Lima-Costa MF, Veras R. Saúde pública e envelhecimento [Editorial]. Cad Saúde Pública 2003; 19:700-1.
9. Freeborn DK, Pope CR, Davis MA, Mullooly JP. Health status, socioeconomic status and utilization of outpatient services for members of a prepaid group practice. Med Care 1977; 15:115-28.

10. Travassos C, Fernandes C, Perez M. Desigualdades sociais, morbidade e consumo de serviços de saúde no Brasil. Série Estudos: Política, Planejamento e Gestão em Saúde 1995; 4:5-26.

11. Almeida CM, Travassos C, Porto S, Labra ME. Health sector reform in Brazil: a case study of inequity. Int J Health Serv 2000; 30:129-62.

12. Travassos C, Viacava F, Fernandes C, Almeida CS. Desigualdades geográficas e sociais na utilização de serviços de saúde no Brasil. Ciênc Saúde Coletiva 2000 ; 5:133-49.

13. Mendoza-Sassi R, Béria JU, Barros AJD. Outpatient health services utilization and associated factors: a population-based study. Rev Saúde Pública 2003; 37:373-8.

14. Ezzati M, Lopez AD, Rodgers A, Vander Hoorn S Murray CJ; Comparative Risk Assessment Collaborating Group. Selected major risk factors and global and regional burden of disease. Lancet 2002; 360:1347-60.

Recebido em 15/Mai/2005

Versão final reapresentada em 08/Mai/2006

Aprovado em 15/Mai/2006 